\title{
A synbiotic combination of Lactobacillus gasseri 505 and Cudrania tricuspidata leaf extract prevents hepatic toxicity induced by colorectal cancer in mice
}

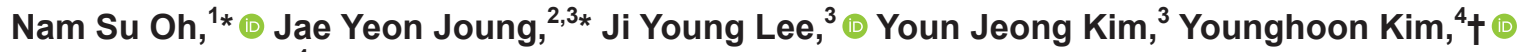 \\ and Sae Hun $\mathrm{Kim}^{4}+$ (i) \\ ${ }^{1}$ Department of Food and Biotechnology, Korea University, Sejong 30019, Korea \\ ${ }^{2}$ R\&D Center, Seoul Dairy Cooperative, Ansan, Kyunggi 425-839, Korea \\ ${ }^{3}$ Department of Biotechnology, College of Life Sciences and Biotechnology, Korea University, Seoul, 02841, Korea \\ ${ }^{4}$ Department of Agricultural Biotechnology and Research Institute of Agriculture and Life Science, Seoul National University, Seoul 08826, Korea
}

\section{ABSTRACT}

Colorectal cancer (CRC) is known to be a lifethreatening disease and commonly leads to metastasis in the liver. Fermented milk acts as an effective carrier for probiotic strains, whose consumption improves host health. Our previous study indicated that fermented milk that included a synbiotic combination of Lactobacillus gasseri 505 (505) and Cudrania tricuspidata leaf extract $(\mathrm{CT})$ resulted in significantly greater anti-oxidative effects than fermented milk without CT. Therefore, we hypothesized that fermented milk containing $\mathrm{CT}$ and 505 (FCT) could result in hepatoprotective effects against $\mathrm{CRC}$-induced liver metastasis. Liver inflammation and CRC were induced in male C57BL/6J mice, using azoxymethane/dextran sodium sulfate, and 505, CT, and FCT were administered to the 3 sample-treated 505, CT, and FCT groups, respectively, for $10 \mathrm{wk}$. The results showed that FCT treatment significantly reduced serum aspartate aminotransferase and alanine aminotransferase concentrations and elevated albumin concentrations. Moreover, the results of histological analysis showed that hepatic steatosis was notably reduced in the FCT group. Among the 3 sample-treated groups, the expression of mRNA associated with enzymes showing anti-oxidative activities, such as superoxide dismutase, catalase, and glutathione reductase, was the highest in the FCT-treated mice. In addition, FCT administration resulted in the greatest anti-inflammatory activity, as inflammatory marker levels (i.e., tumor necrosis factor- $\alpha$, cyclooxygenase-2, myeloperoxidase, and nuclear factor kappa-light-

\footnotetext{
Received August 5, 2019.

Accepted November 19, 2019.

*These authors contributed equally to this study.

†Corresponding authors: ykeys2584@snu.ac.kr and saehkim@korea ac.kr
}

chain enhancer of activated B cells) were significantly downregulated at the mRNA level and the expression of proteins associated with the nuclear factor kappalight-chain enhancer of activated B cells and mitogenactivated protein kinase signaling pathways was suppressed by FCT. Therefore, this study demonstrated that fermented milk containing novel synbiotics has the potential to prevent hepatic toxicity induced because of CRC owing to its enhanced anti-oxidative and antiinflammatory activities.

Key words: colitis-associated colorectal cancer, hepatic toxicity, Cudrania tricuspidata, Lactobacillus gasseri, synbiotic

\section{INTRODUCTION}

Colorectal cancer (CRC), one of the leading causes of which includes inflammatory bowel disease, is the fourth most common cause of cancer-related deaths worldwide (Terzić et al., 2010). Colorectal cancer is strongly associated with chronic inflammation, which could occur from the earliest stage of tumor onset. Azoxymethane (AOM), a metabolite of 1,2-dimethylhydrazine, is a colon cancer-inducing agent that can be used as a tool to evaluate colon tumors in rodents (Chan et al., 2006). It has been reported that the liver and lungs are the most affected organs when colon cancer is induced by AOM (Chan et al., 2006), and the most frequent site of metastasis for CRC is the liver (Valderrama-Trevino et al., 2017). Moreover, the liver plays an important role in the regulation of different physiological activities in the body and participates to a maximum extent in the detoxification of different drugs and xenobiotics (Sharma et al., 2011). The loss of liver capacity impedes the functioning of various organs because of the release of harmful components from the injured liver and the loss of key hepatic detoxifying pathways (Deng et al., 2012). 
In our previous study, Cudrania tricuspidata leaf extract (CT), which is a new plant-based prebiotic source, successfully enhanced the growth and metabolism of Lactobacillus gasseri in milk (Oh et al., 2016a,b, 2019). The supplementation of milk with CT and selected L. gasseri strains had been shown to improve antioxidant activity through their synbiotic interaction (Oh et al., 2016b). Moreover, we found that the administration of L. gasseri 505 (505), CT, and fermented CT-supplemented milk with 505 (FCT) protected the colon against colorectal neoplasia in AOM/dextran sodium sulfate (DSS)-induced CRC mice, via the suppression of pro-inflammatory signals, enhancement of cell cycle arrest, cancer cell apoptosis, intestinal barrier functioning, and inhibition of $\beta$-catenin accumulation. However, the preventive effects of these samples associated with the CRC-derived hepatic disorders have not been explored yet.

Therefore, we aimed to investigate the hepatoprotective effect of FCT, as compared with that observed with the use of 505 or CT alone on colitis-associated CRC, induced by AOM/DSS in mice. To understand the possible mechanisms of action, we examined several markers of liver function, oxidative stress, and inflammation involved in the development of CRC-associated hepatic toxicity.

\section{MATERIALS AND METHODS}

\section{Preparation of C. tricuspidata Leaf Extract}

Fresh C. tricuspidata leaves were purchased from a local market (Sunchang, Jeollabuk-Do, South Korea). About $100 \mathrm{~g}$ of leaves was washed and soaked in $1 \mathrm{~L}$ of distilled water in a shaking water bath, at $100^{\circ} \mathrm{C}$ for 9 h. Then, the leaf extract was filtered using filter paper. The clear solution was concentrated by evaporation and freeze-dried for fermented milk production.

\section{Bacterial Strain}

The probiotic strain L. gasseri 505, originally isolated from human infant feces, was obtained from the Korean Culture Center of Microorganisms (Seoul, South Korea, KCCM 11766P). The probiotic potential of this bacterial isolate was determined by various tests, such as those for acid and bile tolerance, bacterial adhesion capacity, anti-bacterial activity, and cholesterolreducing ability (Oh et al., 2016c). In addition, the $16 \mathrm{~S}$ rRNA gene sequence for the KCCM 11766P bacterial strain has been deposited in the National Center for Biotechnology Information GenBank (http://www.ncbi .nlm.nih.gov/genbank/) and is accessible through accession number KU517710. The strain was sub-cultured 3 times in de Man, Rogosa, and Sharpe broth (Difco Laboratories, Detroit, MI) at $37^{\circ} \mathrm{C}$ for $18 \mathrm{~h}$ under anaerobic conditions before use.

\section{Fermentation of CT-Supplemented Milk}

To evaluate the synbiotic effects of CT leaf extract and 505, CT-supplemented milk was fermented with 505 . The powdered CT leaf extract $[0.2 \%$ (wt/wt)] was added to pre-warmed milk (Seoul Dairy Cooperative, Gyeonggi-do, South Korea), and the mixture was pasteurized at $85^{\circ} \mathrm{C}$ for $15 \mathrm{~min}$ and cooled to $41^{\circ} \mathrm{C}$. Then, the pasteurized milk containing the $\mathrm{CT}$ leaf extract was inoculated with a $3 \%(\mathrm{vol} / \mathrm{vol}$ ) suspension of 505 (approximately $10^{7} \mathrm{cfu} / \mathrm{mL}$ ). The mixture was incubated at $41^{\circ} \mathrm{C}$ for $40 \mathrm{~h}$. Subsequently, the fermented product was freeze-dried and stored at $-20^{\circ} \mathrm{C}$.

\section{Animal Experiments}

Eight-week-old male C57BL/6 mice were purchased from Samtaco Bio Korea (Osan, South Korea). The animals were maintained at $22 \pm 2^{\circ} \mathrm{C}$ and relative humidity $55 \pm 5 \%$, under a 12 -h light/dark cycle. Mice were provided feed (AIN-76A, DooYeol Biotech, Seoul, South Korea) and tap water ad libitum. After a 7-d adaptation period, mice were randomly allocated to the following 5 groups $(\mathrm{n}=10)$ : the untreated control group $(\mathbf{C O N}), \mathrm{AOM}$ and DSS plus PBS-treated group (AOM/DSS), AOM/DSS plus 505 treated group $\left(10^{8} \mathrm{cfu} / \mathrm{kg}\right.$ per day; LG), AOM/DSS plus CT leaf extract-supplemented milk-treated group $(1,500 \mathrm{mg} / \mathrm{kg}$ per day; CT), and AOM/DSS plus CT leaf extractsupplemented milk fermented with the 505-treated group $(1,500 \mathrm{mg} / \mathrm{kg}$ per day; FCT). The additional inflammatory stimulus of DSS in conjugation with $\mathrm{AOM}$ results in tumor development that is restricted to the colon of mice (Vendramini-Costa and Carvalho, 2012). Mice were intraperitoneally injected with $10 \mathrm{mg} /$ $\mathrm{kg}$ AOM (Sigma-Aldrich, St. Louis, MO) on the first day of the experiment and 2.5\% DSS (Sigma-Aldrich) was added to their drinking water for a week, after which they received regular drinking water for $2 \mathrm{wk}$ for their recovery. This cycle was repeated 3 times. Treatments were orally administered to the 3 sample treatment groups (LG, CT, and FCT) for $10 \mathrm{wk}$. The overall experimental schedule is depicted in Figure 1A. The experiment involving mice was approved by the institutional animal care and use committee of Korea University (Seoul, South Korea). 


\section{Histopathological Analysis}

Pathological histology was evaluated according to previously described methods (Oh et al., 2015). After the removal of the liver, tissues were fixed in $10 \%$ neutral-buffered formalin (Sigma-Aldrich). The formalinfixed hepatic tissue samples were processed for histological analysis. Paraffin-embedded tissue sections were stained with hematoxylin and eosin. The tissue samples were then examined and photographed under a light microscope to determine the histopathological changes.

\section{Serum Analysis}

Blood samples were immediately collected in BD Vacutainer SST II Advance (Becton Dickinson, Franklin Lakes, NJ) by cardiac puncture after mice were killed, and the samples were stored at $-20^{\circ} \mathrm{C}$ for $30 \mathrm{~min}$. After centrifugation at $805 \times g$ for $15 \mathrm{~min}$, the serum was transferred to new tube for further analysis. Serum concentrations of aspartate aminotransferase (AST), alanine aminotransferase (ALT), and albumin were measured using AST, ALT, and Albumin Infinity Enzymatic Assay Kits (Thermo Scientific, Waltham, MA), respectively, according to the instructions provided by the manufacturer (https://www.fishersci.com). All measurements were made in triplicate.

\section{Quantitative Real-Time PCR}

Total RNA was extracted from liver tissues using TRIzol reagent (Invitrogen, Carlsbad, CA), according to the instructions provided by the manufacturer. The RNA was quantified using a NanoDrop 2000 spectrophotometer (Thermo Scientific) before cDNA synthesis, using a High Capacity cDNA Reverse Transcription Kit (Applied Biosystems, Foster City, CA). The cDNA samples were stored at $-20^{\circ} \mathrm{C}$ until further use. Then, quantitative real-time PCR (CFX Connect Real-Time PCR Detection System, Bio-Rad, Hercules, CA) was used to detect mRNA expression using the KAPA SYBR FAST qPCR kit universal master mix $(2 \times$; Kapa Biosystems, Boston, MA). The reaction protocol included steps such as denaturation, which was performed at $95^{\circ} \mathrm{C}$ for $3 \mathrm{~min}$, followed by 40 cycles of annealing, performed at $95^{\circ} \mathrm{C}$ for $20 \mathrm{~s}$ and $60^{\circ} \mathrm{C}$ for 40 $\mathrm{s}$, and extension, which was performed at the primer melting temperature for $60 \mathrm{~s}$. The relative gene expression levels were calculated using the comparative threshold cycle method, and values were normalized

A

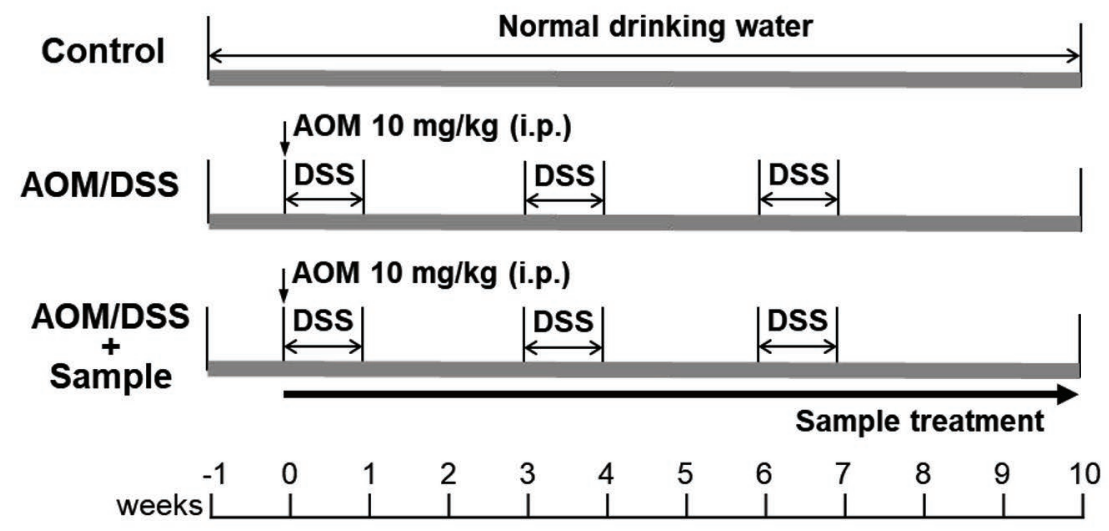

B

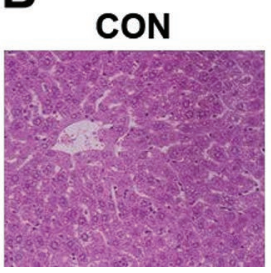
AOM/DSS

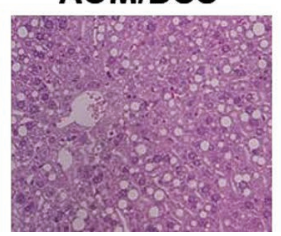

LG

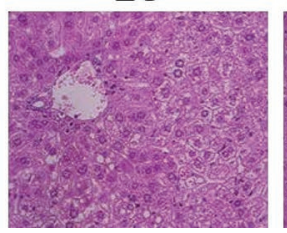

CT

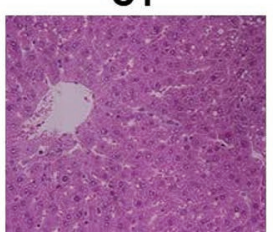

FCT

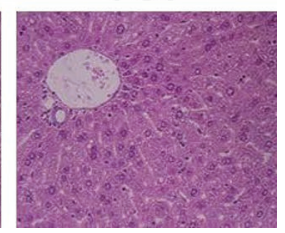

Figure 1. (A) Experimental protocol for colorectal cancer model. Following an azoxymethane (AOM; $10 \mathrm{mg} / \mathrm{kg}$ ) single intraperitoneal (i.p.) injection, mice received $2.5 \%$ dextran sodium sulfate (DSS) in free drinking water for 1 wk, followed by 2 wk of regular drinking water for recovery. The $2.5 \%$ drinking water cycle was repeated 3 times. The LG, CT, and FCT treatment groups were orally administered each sample for 10 wk. (B) Representative photomicrographs depict histological sections of mice liver stained with hematoxylin and eosin, $\times 400$ magnification. $\mathrm{CON}=$ untreated normal control mice; AOM/DSS = mice treated with AOM/DSS; LG = mice treated with AOM/DSS + Lactobacillus gasseri $505\left(10^{8} \mathrm{cfu} / \mathrm{kg}\right.$ per day $) ; \mathrm{CT}=$ mice treated with AOM/DSS + Cudrania tricuspidata leaf extract-supplemented milk (1,500 mg/kg per day); $\mathrm{FCT}=$ mice treated with AOM/DSS + C. tricuspidata leaf extract-supplemented milk fermented with L. gasseri 505 (1,500 mg/kg per day). 
with those for $G A P D H$. The primer sequences of superoxide dismutase $(S O D)$, catalase $(C A T)$, and glutathione reductase $(G R)$, and glutathione peroxidase $(G P x)$ that were associated with an anti-oxidative activity and those of tumor necrosis factor- $\alpha$ (TNFA), cyclooxygenase-2 (COX2), myeloperoxidase $(M P O)$, and nuclear factor kappa-light-chain enhancer of activated B cells $(N F K B)$, which were associated with anti-inflammatory activity, are listed in Supplemental Table S1 (https:// doi.org/10.3168/jds.2019-17411). All samples were run in triplicate.

\section{Western Blot Analysis}

The total protein concentration of the liver homogenate supernatant was determined using a BCA Protein Assay Kit (Thermo Scientific). Protein samples (10 $\mu \mathrm{g})$ were fractionated using 12\% SDS-PAGE and transferred onto a polyvinylidene difluoride membrane, using the Trans-Blot Turbo Transfer System (Bio-Rad). The membranes were blocked with $5 \%$ skim milk in wash buffer (0.1\% Tween-20 in TBS) for $1 \mathrm{~h}$ and then incubated with the primary antibody overnight at $4^{\circ} \mathrm{C}$. Then, the blots were incubated with the appropriate horseradish peroxidase-conjugated secondary antibody for $1 \mathrm{~h}$ at room temperature. The antibodies used in this study are listed in Supplemental Table S2 (https: //doi.org/10.3168/jds.2019-17411). After washing, the membranes were visualized using enhanced chemiluminescence substrate (Bio-Rad) and imaged using the Bio-Rad ChemiDoc MP imaging system. Western blot bands were quantified using ImageJ software (National Institutes of Health, Bethesda, MD), and values were normalized with those of GAPDH.

\section{Statistical Analysis}

All data are expressed in terms of the mean \pm standard deviation. Statistical significance was assessed by Duncan's multiple range tests. The SAS software version 9.2 (SAS Institute Inc., Cary, NC) was used to perform all statistical tests. A $P$-value $<0.05$ was considered statistically significant.

\section{RESULTS}

\section{Hepatic Histopathology}

The histopathological analysis of liver sections showed changes due to AOM/DSS and sample treatment (Figure 1A). Treatment with AOM/DSS led to a significant increase of fatty infiltration into the hepatocytes, as compared with that observed for the CON group. Fatty infiltration was visualized as white spots on the histological section of the liver, which were associated with an increased risk of cancer. However, the size and number of fatty spots were significantly decreased in the 505, CT, and FCT groups. Particularly, marked recovery was observed in the FCT group.

\section{Hepatic Biochemical Variables}

To determine the effect of AOM/DSS and samples on liver function, liver function tests were performed through the analysis of serum. Mice with CRC induced by AOM/DSS showed impaired liver functioning, and this was demonstrated by a significant increase in serum AST and ALT concentrations, as compared with those in CON mice (Table 1). On the other hand, the administration of 505, CT leaf extract, and FCT reduced AOM/DSS-induced changes in the serum concentrations of AST and ALT. The FCT especially normalized the serum levels to those of the CON group. However, serum albumin had the opposite expression pattern to AST and ALT. The AOM/DSS tended to cause a decrease in the concentration of serum albumin, whereas the concentration of serum albumin was recovered in the LG, CT, and FCT groups.

\section{Effect of FCT on the mRNA Expression of Oxidative Stress Markers in the Liver}

We investigated the effect of samples on the expression of oxidative stress regulatory genes in the liver of AOM/DSS-induced CRC mice. The AOM/DSS group exhibited a significant decrease in the levels of antioxidative related markers, such as $S O D, C A T$, and $G R$, as compared with those of the CON group (Figure 2). The administration of FCT remarkably inhibited the AOM/DSS-induced decrease in the mRNA levels. Although treatment with 505 and CT leaf extract tended to increase the expression of genes associated with antioxidative effects in mice from the AOM/DSS group, the recorded effect was insignificant. However, no significant changes were observed in glutathione peroxidase $(G P x)$ expression, after treatment with AOM/DSS and samples had been provided.

\section{Effect of FCT on Hepatic Inflammation in CRC-Induced Mice}

Quantitative RT-PCR and Western blot analysis were conducted to measure the expression of mRNA and protein related to the hepatic inflammation in the AOM/DSS-induced CRC mice. The administration of AOM/DSS into mice significantly upregulated the mRNA levels of inflammatory markers in the liver, such as TNFA, COX2, MPO, and NFKB (Figure 3). On the 
Table 1. Liver function markers in experimental mice ${ }^{1,2}$

\begin{tabular}{|c|c|c|c|c|c|}
\hline Item & $\mathrm{CON}$ & $\mathrm{AOM} / \mathrm{DSS}$ & $\mathrm{LG}$ & $\mathrm{CT}$ & FCT \\
\hline $\operatorname{ALT}(\mathrm{U} / \mathrm{L})$ & $125.40 \pm 2.97^{\mathrm{ab}}$ & $151.00 \pm 10.41^{\mathrm{c}}$ & $135.00 \pm 6.24^{\mathrm{b}}$ & $134.75 \pm 6.70^{\mathrm{b}}$ & $123.00 \pm 6.78^{\mathrm{a}}$ \\
\hline
\end{tabular}

${ }^{\mathrm{a}-\mathrm{c}}$ Mean values in a row with different superscripts are significantly different as determined by Tukey's test $(P<0.05)$.

${ }^{1}$ Values are expressed as the mean $\pm \mathrm{SD}(\mathrm{n}=16)$.

${ }^{2} \mathrm{AST}=$ aspartate aminotransferase; ALT $=$ alanine aminotransferase $; \mathrm{CON}=$ untreated normal control mice; AOM $/ \mathrm{DSS}=$ mice treated with azoxymethane $(\mathrm{AOM}) /$ dextran sodium sulfate (DSS); LG = mice treated with AOM/DSS + Lactobacillus gasseri 505 (10 ${ }^{8}$ cfu $/ \mathrm{kg}$ per day); $\mathrm{CT}=$ mice treated with AOM/DSS + Cudrania tricuspidata leaf extract-supplemented milk $(1,500 \mathrm{mg} / \mathrm{kg}$ per day $) ; \mathrm{FCT}=$ mice treated with AOM/DSS + C. tricuspidata leaf extract-supplemented milk fermented with L. gasseri 505 (1,500 mg/kg per day).

other hand, the hepatic mRNA levels of TNFA, NFKB, $C O X 2$, and $M P O$ were significantly decreased in AOM/ DSS-treated mice after the oral consumption of 505, CT leaf extract, and FCT. The decreased mRNA levels were similar to those observed in $\mathrm{CON}$ mice. Moreover, AOM/DSS-induced CRC mice exhibited significantly increased protein levels of inflammatory markers such as p50, p65, and phosphorylated p105 (p-p105), which were correlated with the levels observed during the NF- $\kappa$ B-I $\kappa$ B pathway (Figure 4). As shown in Figure 4 , the treatment of samples upregulated the levels of mitogen-activated protein kinase (MAPK) related proteins [i.e., extracellular signal-regulated kinase (ERK1/2), phosphorylated ERK1/2 (p-ERK1/2),
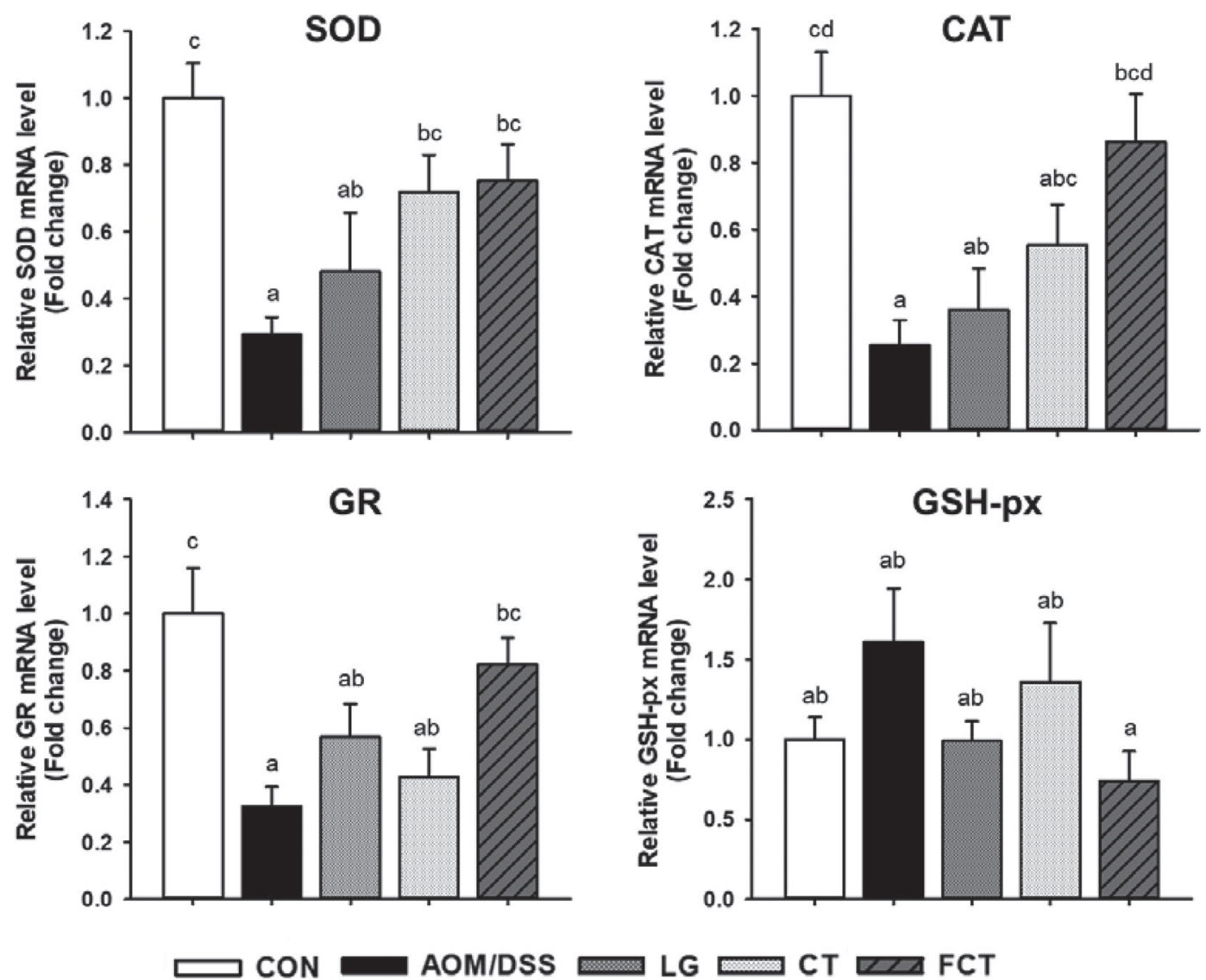

Figure 2. Effect of fermented milk containing Cudrania tricuspidata leaf extract and Lactobacillus gasseri 505 on liver mRNA expression of oxidative stress-related markers in azoxymethane (AOM)/dextran sodium sulfate (DSS)-induced colorectal cancer mice. The mRNA levels of superoxide dismutase $(S O D)$, catalase $(C A T)$, glutathione peroxidase $(G P x)$, and glutathione reductase $(G R)$ were measured by real-time PCR and normalized with respect to the mRNA level of $G A P D H$. Results are presented as the mean \pm SD. Different letters indicate significant differences between the different groups $(P<0.05)$. CON $=$ untreated control mice; AOM/DSS $=$ mice treated with AOM/DSS; LG $=$ mice treated with AOM/DSS + Lactobacillus gasseri $505\left(10^{8} \mathrm{cfu} / \mathrm{kg}\right.$ per day $) ; \mathrm{CT}=$ mice treated with AOM/DSS + Cudrania tricuspidata leaf extractsupplemented milk $(1,500 \mathrm{mg} / \mathrm{kg}$ per day); FCT $=$ mice treated with AOM/DSS + C. tricuspidata leaf extract-supplemented milk fermented with L. gasseri 505 (1,500 mg/kg per day). 

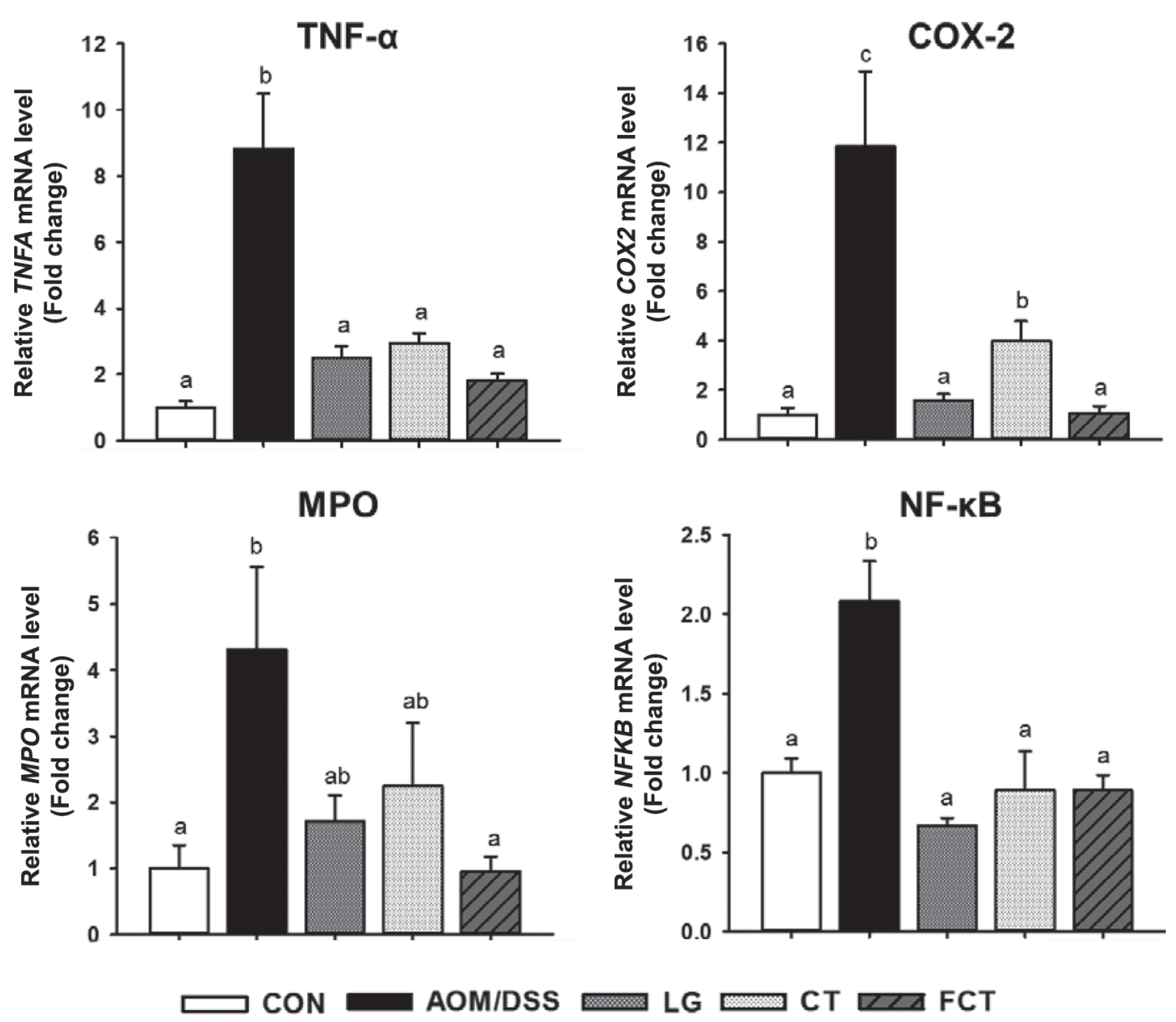

Figure 3. Effect of fermented milk containing Cudrania tricuspidata leaf extract and Lactobacillus gasseri 505 on liver mRNA expression of inflammatory-related markers in azoxymethane (AOM)/dextran sodium sulfate (DSS)-induced colorectal cancer mice. The mRNA levels of tumor necrosis factor- $\alpha$ (TNFA), nuclear factor kappa-light-chain enhancer of activated B cells (NFKB), cyclooxygenase-2 (COX2), and myeloperoxidase $(M P O)$ were measured by real-time PCR and normalized with respect to the mRNA level of GAPDH. Results are presented as the mean $\pm \mathrm{SD}$. Different letters indicate significant differences between the different groups $(P<0.05)$. CON $=$ untreated control mice; AOM $\mathrm{DSS}=$ mice treated with AOM/DSS; LG = mice treated with AOM/DSS + Lactobacillus gasseri $505\left(10^{8}\right.$ cfu $/ \mathrm{kg}$ per day); CT $=$ mice treated with AOM/DSS + Cudrania tricuspidata leaf extract-supplemented milk $(1,500 \mathrm{mg} / \mathrm{kg}$ per day); FCT $=$ mice treated with AOM/DSS $+C$. tricuspidata leaf extract-supplemented milk fermented with L. gasseri 505 (1,500 mg/kg per day).

c-jun N-terminal kinase (JNK), phosphorylated JNK (p-JNK), p38, and phosphorylated p38 (p-p38)]. In contrast, the administration of samples tended to decrease the expression of these proteins. The FCT especially showed the greatest anti-inflammatory effects, based on its suppression of inflammatory markers.

\section{DISCUSSION}

Previously, we reported that the synbiotic combination of 505 and CT had greater anti-oxidative properties than those observed with 505 or $\mathrm{CT}$ alone (Oh et al., 2016c). Additionally, FCT exhibited protective effects against colon neoplasia and inflammation by enhancing the activation of cell cycle arrest, cancer cell apoptosis, and intestinal barrier functioning in AOM/ DSS-induced CRC mice. However, the preventive effects of FCT on the CRC-derived hepatic disorders, known to be associated with liver metastasis, have not been reported. The liver is the organ where the distant metastasis of CRC occurs most frequently due to its portal circulation (Valderrama-Trevino et al., 2017). The results of this study indicated that FCT had a positive effect on the prevention of hepatotoxicity occurring because of AOM/DSS-induced CRC, in accordance with the regulatory effects of FCT on oxidative stress and inflammation-related markers in AOM/DSS-treated mice. Azoxymethane is a colon carcinogen that acts as a tumor-inducing agent, and in combination with DSS, it results in tumor development in mice (De Robertis et al., 2011). Moreover, a previous study reported that AOM-treated mice developed liver injury, characterized histologically by extensive fatty degeneration, necrosis, inflammatory cell infiltration, and other histological 
manifestations (Abdella et al., 2016). In this study, we identified the effect of AOM/DSS-induced CRC on hepatic damage through histopathology analysis. The results showed that hepatic steatosis was significantly aggravated by AOM/DSS treatment, whereas the administration of FCT caused the recovery of the hepatic histological status to a level that was similar to that of the CON group. Liver function was normalized by FCT, as shown by the results of histological analysis and serum analysis of ALT, AST, and albumin. The elevated concentrations of ALT and AST are generally used as a diagnostic indicator of hepatocyte damage (Rajesh and Latha, 2004); for example, increased serum levels indicate the occurrence of intrahepatic cholestasis (Siddique and Kowdley, 2012).

The hepatoprotective mechanism involves the ability of NF- $\kappa \mathrm{B}$ to maintain antioxidant defenses, by controlling the expression of several key reactive oxygen species-scavenging proteins (Pham et al., 2004; Kamata et al., 2005). Excessive reactive oxygen species accumulation promotes cell death through various mechanisms, including prolonged JNK activation (Kamata et al., 2005). Increased levels of JNK phosphorylation and kinase activity were observed in the livers of Ikk $\gamma^{\text {hhep }}$ mice and diethylnitrosamine-phenobarbital-challenged $\operatorname{Ikk} \beta^{\Delta \text { hep }}$ mice (Maeda et al., 2005; Luedde et al., 2007).

A
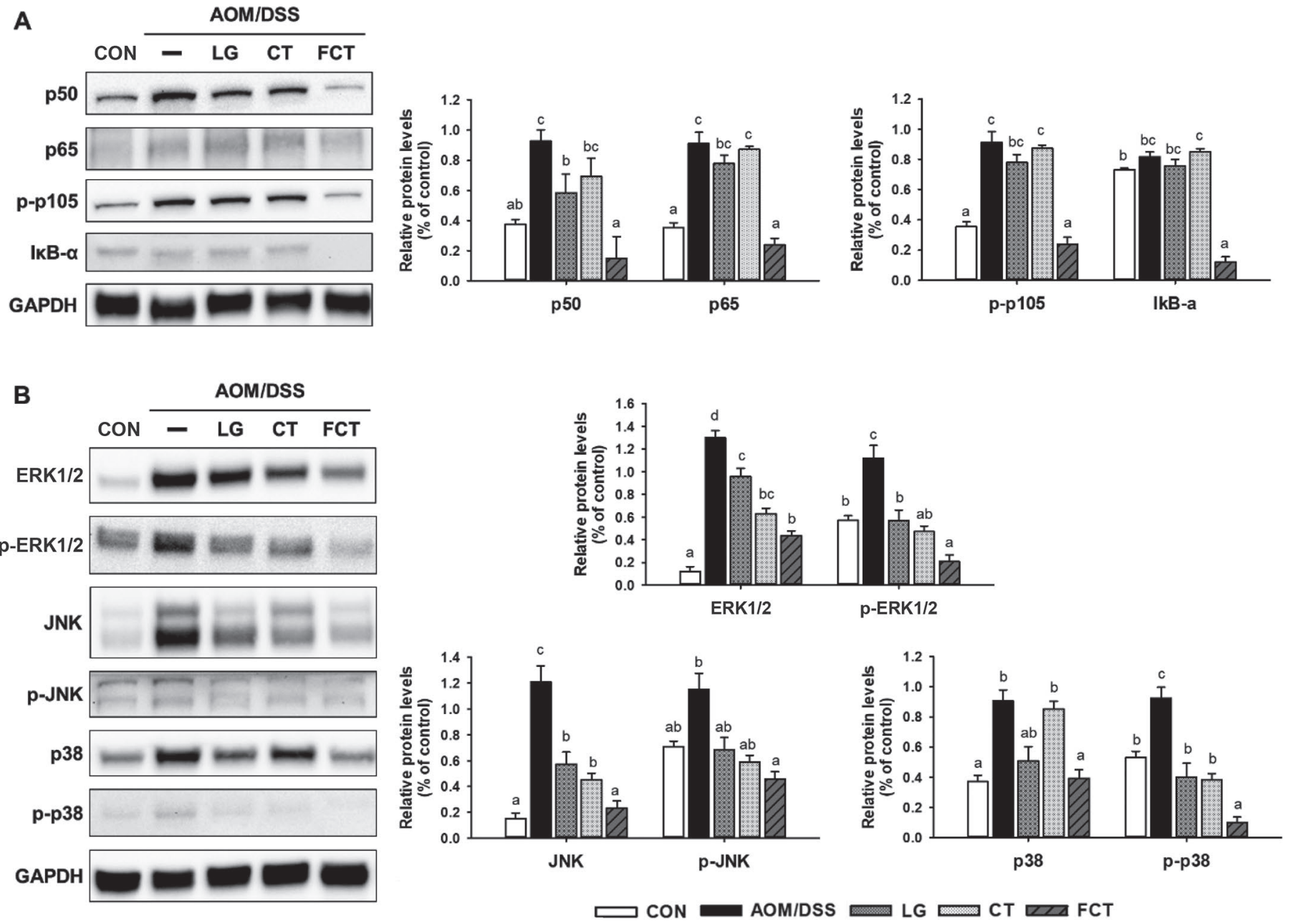

Figure 4. Effects of fermented milk containing Cudrania tricuspidata leaf extract and Lactobacillus gasseri 505 on (A) nuclear factor kappalight-chain enhancer of activated B cells (NF- $\mathrm{B}$ ) and (B) mitogen-activated protein kinase (MAPK) signaling in liver of azoxymethane (AOM)/ dextran sodium sulfate (DSS)-induced colorectal cancer mice. The NF- $\kappa$ B and MAPK signaling molecules were measured by Western blot analysis and quantified after normalization to GAPDH. Results are presented as the mean \pm SD. Different letters indicate significant differences between the different groups $(P<0.05)$. CON $=$ untreated control mice; AOM $/$ DSS $=$ mice treated with AOM $/$ DSS; LG $=$ mice treated with $\mathrm{AOM} / \mathrm{DSS}+$ Lactobacillus gasseri $505\left(10^{8} \mathrm{cfu} / \mathrm{kg}\right.$ per day); CT = mice treated with AOM/DSS + Cudrania tricuspidata leaf extract-supplemented milk $(1,500 \mathrm{mg} / \mathrm{kg}$ per day $) ; \mathrm{FCT}=$ mice treated with AOM/DSS $+C$. tricuspidata leaf extract-supplemented milk fermented with $L$. gasseri 505 (1,500 mg/kg per day). p-p105 = phosphorylated p105; ERK1/2 = extracellular signal-regulated kinase; p-ERK1 $/ 2=$ phosphorylated ERK1 $/ 2 ; \mathrm{JNK}=$ c-jun N-terminal kinase; p-JNK = phosphorylated JNK; p-p38 = phosphorylated p38. 
In the current study, the administration of FCT into AOM/DSS-induced CRC mice significantly increased the expression of anti-oxidative activity-related genes, including SOD, CAT, and GR, as compared with the levels for the mice in the AOM/DSS group that were not given FCT.

Inflammatory stimuli such as TNF, IL-1 $\beta$, and LPS

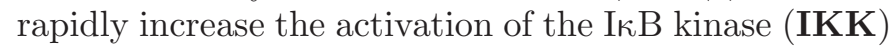
complex, which is composed of the IKK $\alpha$, IKK $\beta$, and IKK $\gamma$ subunits, which results in I $\mathrm{B}$ phosphorylation and NF- $\kappa$ B activation (Ghosh and Karin, 2002). The $\mathrm{NF}-\kappa \mathrm{B}$ regulates a variety of genes, including cytokines (e.g., IL1, IL2, IL6, IL12, and TNFA), chemokines [e.g.,

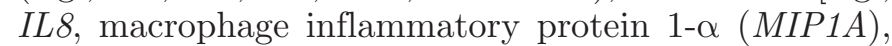
and monocyte chemoattractant protein-1 (MCP1)], adhesion molecules [e.g., intercellular adhesion molecule-1 (ICAM1), vascular cell adhesion molecule-1 (VCAM1), and E-selectin $(S E L E)]$, acute phase proteins [e.g., serum amyloid A $(S A A)]$, and inducible effector enzymes [e.g., inducible nitric oxide synthase and COX2; Ghosh and Karin, 2002]. Expression of COX-2 is induced by a variety of pro-inflammatory stimuli, and COX-2 is a crucial enzyme involved in the biosynthesis of prostaglandins. In the liver, COX-2 and prostaglandin production has been implicated in inflammation, matrix remodeling, progress of fibrosis, and the development of hepatocellular carcinoma (Martinez-Manez and Sancenón, 2003). The increased MPO activity is often used as a marker for the infiltration of neutrophils into tissues, and indicates the occurrence of inflammation (Haqqani et al., 1999). Genes regulating inflammation, such as TNFA, NFKB, COX2, and MPO in the liver of AOM/DSS-treated mice, were upregulated, whereas FCT administration normalized the expression of those genes; these results were in agreement with those reported in previous studies. The NF- $\kappa \mathrm{B}$ was first identified as a group of dimeric transcription factors, based on their interaction with the immunoglobulin lightchain enhancer in B cells (Sen and Baltimore, 1986). Protein factors include NF- $\kappa$ B1 (p105 and p50), NF$\kappa$ B2 (p100 and p52), RelA (p65), RelB, and c-Rel. In nonstimulated cells, most NF- $\kappa \mathrm{B}$ dimers are retained in the cytoplasm because they bind to inhibitory $\mathrm{I} \kappa \mathrm{B}$ proteins, except for the dimers formed by p105 and p100, which are inactive and contain intrinsic IкB-like-

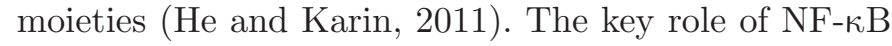
in liver homeostasis was first revealed by the study of RelA/p65-deficient mice, which exhibit embryonic lethality with extensive liver apoptosis and degeneration (Beg et al., 1995; Ma et al., 2011). In the present study, AOM/DSS stimulated the expression of p50, $\mathrm{p} 65$, p-p105, and $\mathrm{I} \kappa \mathrm{B} \alpha$ in the hepatic tissue, whereas the expression of those proteins was suppressed in $\mathrm{FCT}$ group, which resulted in the inhibition of NF- $\kappa \mathrm{B}$ ac- tivation. Furthermore, NF- $\kappa$ B plays a central role in the overall generation of the immune response, through its ability to regulate apoptosis and cell proliferation (Karin and Ben-Neriah, 2002), which is highly associated with the MAPK family. To date, at least 3 major MAPK cascades have been found to be involved in the activation of ERK, JNK/stress-activated kinases, and p38 MAPK (Ma et al., 2014). Among these, ERK are activated by various cytokines and growth factors, and play a central role in cell growth and differentiation. The JNK and p38 MAPK are preferentially activated by pro-inflammatory cytokines such as TNF- $\alpha$, in addition to environmental and genotoxic stress (Nakagawa and Maeda, 2012a). After activation, stress-activated MAPK phosphorylate specific serine/threonine residues of target substrates and are involved in a variety of cellular functions, such as cell death, survival, proliferation, migration, and inflammation (Nakagawa and Maeda, 2012b). Our results suggest that FCT could attenuate the hepatotoxicity induced by CRC through suppressing the overexpression of inducible nitric oxide synthase and COX2, which had been induced by AOM/ DSS. Moreover, FCT regulated the expression of ERK, p-ERK, JNK, p-JNK, p38, and p-p38, leading to the prevention of liver toxicity by the modulation of inflammatory cytokines.

Consequently, 2 important findings were obtained in this study. First, the results suggested that AOM/ DSS treatment induced hepatic steatosis and inflammation, as well as CRC. Second, the novel combination of 505 and $\mathrm{CT}$, which was achieved by the fermentation of CT-supplemented milk with 505, regulated anti-oxidative and inflammatory markers, including NF- $\kappa \mathrm{B}$ and MAPK signaling pathway-related proteins. Moreover, these findings help us to understand the detailed mechanism by which colon-liver inflammation occurs. In addition, our data support the use of milk as a high-value-added product for dairy and pharmaceutical industries because FCT could be used as a natural prophylactic treatment in patients with colon tumorigenesis to prevent hepatic injury.

\section{ACKNOWLEDGMENTS}

This research was supported by the High Value-Added Food Technology Development Program of the Korea Institute of Planning and Evaluation for Technology in Food, Agriculture, Forestry, and Fisheries (iPET), and the Ministry for Food, Agriculture, Forestry, and Fisheries of the Republic of Korea (Jeollanam-do, Korea, 117069-03-3-SB010) and Korea University Grant (Sejong, Korea, K1915151). The authors have not stated any conflicts of interest. 


\section{REFERENCES}

Abdella, E. M., A. M. Mahmoud, and A. M. El-Derby. 2016. Brown seaweeds protect against azoxymethane-induced hepatic repercussions through up-regulation of peroxisome proliferator-activated receptor gamma and attenuation of oxidative stress. Pharm. Biol. 54:2496-2504. https://doi.org/10.3109/13880209.2016.1160938.

Beg, A. A., W. C. Sha, R. T. Bronson, S. Ghosh, and D. Baltimore. 1995. Embryonic lethality and liver degeneration in mice lacking the RelA component of NF- $\kappa$ B. Nature 376:167-170. https://doi .org/10.1038/376167a0

Chan, C. H., D. Cook, and C. P. Stanners. 2006. Increased colon tumor susceptibility in azoxymethane treated CEABAC transgenic mice. Carcinogenesis 27:1909-1916. https://doi.org/10.1093/carcin/ bgl040.

De Robertis, M., E. Massi, M. L. Poeta, S. Carotti, S. Morini, L. Cecchetelli, E. Signori, and V. M. Fazio. 2011. The AOM/DSS murine model for the study of colon carcinogenesis: From pathways to diagnosis and therapy studies. J. Carcinog. 10:9.

Deng, L., X. Gui, Y. Xiong, S. Gao, R. Yang, Y. Rong, J. Hu, and Y. Yan. 2012. End-stage liver disease: Prevalence, risk factors and clinical characteristics in a cohort of HIV-HCV coinfected Han Chinese. Clin. Res. Hepatol. Gastroenterol. 36:574-582. https:// doi.org/10.1016/j.clinre.2012.02.007.

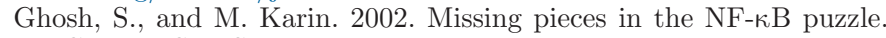
Cell 109:S81-S96.

Haqqani, A. S., R. T. Cowling, J. A. Maroun, and H. C. Birnboim. 1999. Characterization of a polyclonal antibody to human thymidylate synthase suitable for the study of colorectal cancer specimens. J. Histochem. Cytochem. 47:1563-1574. https://doi.org/10 $.1177 / 002215549904701208$.

He, G., and M. Karin. 2011. NF-кB and STAT3-key players in liver inflammation and cancer. Cell Res. 21:159-168. https://doi.org/10 $.1038 /$ cr.2010.183.

Kamata, H., S.-i. Honda, S. Maeda, L. Chang, H. Hirata, and M. Karin. 2005. Reactive oxygen species promote $\mathrm{TNF} \alpha$-induced death and sustained JNK activation by inhibiting MAP kinase phosphatases. Cell 120:649-661. https://doi.org/10.1016/j.cell.2004.12 .041 .

Karin, M., and A. Lin. 2002. NF-kappaB at the crossroads of life and death. Nat. Immunol. 3:221-227. https://doi.org/10.1038/ni0302 -221 .

Luedde, T., N. Beraza, V. Kotsikoris, G. van Loo, A. Nenci, R. De Vos, T. Roskams, C. Trautwein, and M. Pasparakis. 2007. Deletion of NEMO/IKK $\gamma$ in liver parenchymal cells causes steatohepatitis and hepatocellular carcinoma. Cancer Cell 11:119-132. https://doi .org/10.1016/j.ccr.2006.12.016.

Ma, J.-Q., J. Ding, L. Zhang, and C.-M. Liu. 2014. Hepatoprotective properties of sesamin against $\mathrm{CCl} 4$ induced oxidative stress-mediated apoptosis in mice via JNK pathway. Food Chem. Toxicol. 64:41-48. https://doi.org/10.1016/j.fct.2013.11.017.

Ma, J.-Q., C.-M. Liu, Z.-H. Qin, J.-H. Jiang, and Y.-Z. Sun. 2011. Ganoderma applanatum terpenes protect mouse liver against benzo $(\alpha)$ pyren-induced oxidative stress and inflammation. Environ. Toxicol. Pharmacol. 31:460-468. https://doi.org/10.1016/j.etap .2011.02.007.

Maeda, S., H. Kamata, J.-L. Luo, H. Leffert, and M. Karin. 2005. IKK $\beta$ couples hepatocyte death to cytokine-driven compensatory proliferation that promotes chemical hepatocarcinogenesis. Cell 121:977-990. https://doi.org/10.1016/j.cell.2005.04.014.

Martinez-Manez, R., and F. Sancenón. 2003. Fluorogenic and chromogenic chemosensors and reagents for anions. Chem. Rev. 103:4419 4476. https://doi.org/10.1021/cr010421e.

Nakagawa, H., and S. Maeda. 2012a. Inflammation-and stress-related signaling pathways in hepatocarcinogenesis. World J. Gastroenterol. 18:4071-4081. https://doi.org/10.3748/wjg.v18.i31.4071.

Nakagawa, H., and S. Maeda. 2012b. Molecular mechanisms of liver injury and hepatocarcinogenesis: Focusing on the role of stress-ac- tivated MAPK. Patholog. Res. Int. 2012:172894. https://doi.org/ $10.1155 / 2012 / 172894$.

Oh, N. S., K. Kim, S. Oh, and Y. Kim. 2019. Enhanced production of galactooligosaccharides enriched skim milk and applied to potentially synbiotic fermented milk with Lactobacillus rhamnosus 4B15. Food Sci. Anim. Resour. 39:725-741. https://doi.org/10 $.5851 /$ kosfa.2019.e55.

Oh, N. S., J. Y. Lee, J. Y. Joung, K. S. Kim, Y. K. Shin, K. W. Lee, S. H. Kim, S. Oh, and Y. Kim. 2016a. Microbiological characterization and functionality of set-type yogurt fermented with potential prebiotic substrates Cudrania tricuspidata and Morus alba L. leaf extracts. J. Dairy Sci. 99:6014-6025. https://doi.org/10.3168/jds $.2015-10814$

Oh, N. S., J. Y. Lee, and Y. Kim. 2016b. The growth kinetics and metabolic and antioxidant activities of the functional synbiotic combination of Lactobacillus gasseri 505 and Cudrania tricuspidata leaf extract. Appl. Microbiol. Biotechnol. 100:10095-10106. https: //doi.org/10.1007/s00253-016-7863-3.

Oh, N. S., J. Y. Lee, S. Oh, J. Y. Joung, S. G. Kim, Y. K. Shin, K. W. Lee, S. H. Kim, and Y. Kim. 2016c. Improved functionality of fermented milk is mediated by the synbiotic interaction between Cudrania tricuspidata leaf extract and Lactobacillus gasseri strains. Appl. Microbiol. Biotechnol. 100:5919-5932. https://doi.org/10 $.1007 / \mathrm{s} 00253-016-7414-y$.

Oh, N. S., M. R. Park, K. W. Lee, S. H. Kim, and Y. Kim. 2015. Dietary Maillard reaction products and their fermented products reduce cardiovascular risk in an animal model. J. Dairy Sci. 98:5102-5112. https://doi.org/10.3168/jds.2015-9308.

Pham, C. G., C. Bubici, F. Zazzeroni, S. Papa, J. Jones, K. Alvarez, S. Jayawardena, E. De Smaele, R. Cong, C. Beaumont, F. M. Torti, S. V. Torti, and G. Franzoso. 2004. Ferritin heavy chain upregulation by $\mathrm{NF}-\kappa \mathrm{B}$ inhibits $\mathrm{TNF} \alpha$-induced apoptosis by suppressing reactive oxygen species. Cell 119:529-542. https://doi.org/10 $.1016 / j . c e l l .2004 .10 .017$.

Rajesh, M. G., and M. Latha. 2004. Preliminary evaluation of the antihepatotoxic activity of Kamilari, a polyherbal formulation. J. Ethnopharmacol. 91:99-104. https://doi.org/10.1016/j.jep.2003 .12.011.

Sen, R., and D. Baltimore. 1986. Inducibility of $\kappa$ immunoglobulin enhancer-binding protein NF- $\kappa$ B by a posttranslational mechanism. Cell 47:921-928. https://doi.org/10.1016/0092-8674(86)90807-X.

Sharma, S. K., S. M. Arogya, D. H. Bhaskarmurthy, A. Agarwal, and C. C. Velusami. 2011. Hepatoprotective activity of the Phyllanthus species on tert-butyl hydroperoxide (t-BH)-induced cytotoxicity in HepG2 cells. Pharmacogn. Mag. 7:229-233. https://doi.org/10 .4103/0973-1296.84237.

Siddique, A., and K. V. Kowdley. 2012. Approach to a patient with elevated serum alkaline phosphatase. Clin. Liver Dis. 16:199-229. https://doi.org/10.1016/j.cld.2012.03.012.

Terzić, J., S. Grivennikov, E. Karin, and M. Karin. 2010. Inflammation and colon cancer. Gastroenterology 138:2101-2114. https:// doi.org/10.1053/j.gastro.2010.01.058.

Valderrama-Trevino, A. I., B. Barrera-Mera, J. C. Ceballos-Villalva, and E. E. Montalvo-Jave. 2017. Hepatic metastasis from colorectal cancer. Euroasian J. Hepatogastroenterol. 7:166-175. https://doi .org/10.5005/jp-journals-10018-1241.

Vendramini-Costa, D. B., and J. E. Carvalho. 2012. Molecular link mechanisms between inflammation and cancer. Curr. Pharm. Des. 18:3831-3852. https://doi.org/10.2174/138161212802083707.

\section{ORCIDS}

Nam Su Oh ๑ https://orcid.org/0000-0002-0712-7843 Ji Young Lee ๑ https://orcid.org/0000-0003-4838-6128 Younghoon Kim @ (1) https://orcid.org/0000-0001-6769-0657 Sae Hun Kim @ ( https://orcid.org/0000-0002-0990-2268 